\title{
Combining Digital with Analog Circuits in a Core Course for a Multidisci- plinary Engineering Curriculum
}

\section{Dr. Harold R Underwood, Messiah College}

Dr. Underwood received his Ph.D. in Electrical Engineering at the University of Illinois at UrbanaChampaign (UIUC) in 1989, and has been a faculty member of the engineering Department at Messiah College since 1992. Besides teaching Circuits, Electromagnetics, and Communications Systems, he supervises engineering students in the Communications Technology Group on credited work in the Integrated Projects Curriculum (IPC) of the Engineering Department, and other students who participate voluntarily via the Collaboratory for Strategic Parnternships and Applied Research. His on-going projects include improving Flight Tracking and Messaging Systems for small planes in remote locations, and developing an assistive communication technology using Wireless Enabled Remote Co-presence for cognitively and behaviorally challenged individuals including those with high-functioning autism, or PTSD.

\section{Dr. Donald George Pratt, Messiah College}

Dr. Pratt is a Professor of Engineering at Messiah College where he has taught since 1993. Over the past $20+$ years, he has become known for his work with students on an eclectic mix of practical, hands-on projects involving such things as electric vehicles, aircraft, vehicles for use in developing countries, and methods of finding and removing antipersonnel land mines. Dr. Pratt is a co-founder of the Collaboratory for Strategic Partnerships and Applied Research. He and his wife of 30+ years have two grown children and three grandchildren. An avid pilot and builder, he enjoys flying over the beautiful farms and forests of the Cumberland Valley. 


\section{Combining Digital with Analog Circuits in a Core Course for a Multidisciplinary Engineering Curriculum}

A multidisciplinary engineering curriculum requires certain core courses to provide students with the content they will need to be successful in subsequent coursework, projects and beyond. Circuit Analysis, a common core course, has traditionally emphasized the analog side, leaving digital circuits for electrical or computer specializations. While a number of recent papers ${ }^{3-8}$ address improved methods of instruction for Circuit Analysis, strategic ordering of topics and selection of content also makes a difference in preparing students for the curriculum as a whole. With the growing infusion of digital technology in contemporary practice, we believe students in all engineering disciplines should have exposure to digital theory, at least at a basic level. Thus, at Messiah College, we have formed a new Circuits I core course combining introductory analog and digital circuit theory. Accordingly, we replaced our Circuit Analysis and Digital Electronics courses with a new Circuits 1, 2 sequence. While the Circuits 2 course takes up more advanced topics required for electrical and computer specializations, the Circuits 1 course covers basic analog and digital theory, including both discrete circuits and selected integrated circuit devices, working knowledge of which is required for competency in all engineering disciplines. Such competency allows multidisciplinary teams to work together more effectively, when deciding how to implement circuit functionality, make digital measurements, analyze and share digitized data, and plan the flow of information through newly designed systems. This paper provides details on course content division, textbook selection, lecture and lab adjustments, student reaction and other lessons learned, for the benefit of those who wish to try this approach.

\section{Introduction}

A course on electric circuits has long been one of the core courses in a traditional engineering curriculum, providing a basic foundation for students specializing in a variety of disciplines. A typical first semester engineering course on electric circuits such as Circuit Analysis emphasizes linear, discrete elements such as the voltage and/or current source, resistor (R), capacitor (C) and inductor (L), focusing on how to find simplified equivalent circuit models, and solve for circuit related quantities in various component configurations. For practical applications, other electronic devices such as light emitting diodes and operational amplifiers may be introduced, without a detailed analysis of what happens inside the "black box." A traditional treatment begins with circuits having direct current (DC) sources, moves to first and second order time dependent responses of $\mathrm{R}, \mathrm{L}$, and/or $\mathrm{C}$ combinations, and ends with alternating current (AC) circuits. However, the nature and behavior of circuits in such a first course is usually assumed to involve voltages that have a continuous range of possibilities; that is, it has usually been restricted to analog type. 
While the traditional engineering Circuit Analysis course has emphasized the analog rather than digital circuits and devices, the ever increasing infusion of digital technology in all fields of engineering suggests that students in all disciplines need exposure to digital theory and experience with basic digital applications/design. For example, Instrumentation and Measurement, a course traditionally taken primarily by students in the Mechanical concentration, has become increasingly focused on digital methods of making measurements, as mechanical transducers are rapidly being replaced by solid state electronic sensors with integrated linearization, temperature compensation, and often A/D conversion circuitry, usually on the same substrate. Data acquisition and signal processing are now done primarily using digital recordings of data rather than analog signals. To be competent in their field, students like those in the Mechanical concentration who take only one circuits course need to know how to handle digital data. Thus, at Messiah College, we have replaced our Circuit Analysis and Digital Electronics courses with a new Circuits $1 \& 2$ (Circ1, Circ2) sequence. The Circ2 course takes up more advanced topics required for students pursuing the Electrical (Elect) and Computer (Comp) concentrations, whereas the Circ1 course addresses basic circuits, both analog and digital, providing a broader foundation for any engineering concentration. While tradeoffs exist in removing certain topics traditionally offered in the first semester Circuit Analysis course (e.g., RL, RLC, and AC circuit analysis relegated to Circ2), we believe it is more important to make room for the digital circuit content in Circ1, so that all engineering students can be exposed to the digital material. Furthermore, we have observed that students seem to find the digital circuits easier and more fun to learn. Thus, adding digital content to Circ1 appears to help engineering students have a broader and more positive first experience with electric circuits.

Background \& context of the academic curriculum

Transition to the new Circ1, Circ2 course sequence began in our engineering curriculum during spring 2013. In our multidisciplinary engineering program at Messiah College, offering six concentrations, the Circ1 course is typically taken by all students in their second or third semester. In the fourth semester, students in Elect or Comp concentrations follow on to Circ2, taking up the second more advanced half of traditional analog and digital circuit treatment. By that time, these students have completed their Calculus sequence, and normally take Differential Equations concurrently. Overall, no topics are lost in the exchange from the old Circuit Analysis and Digital Electronics to the new Circ1, Circ2 sequence, and Circ2 becomes the pre-requisite for Analog Electronics and Microprocessors in the electrical track. Most importantly for this paper, students on the Elect or Comp tracks do not lose course content by the switch to Circ1 and Circ2; instead, they benefit by the breadth and timing of exposure to digital circuits earlier in their curriculum. 
For students who specialize in concentrations other than Elect or Comp, such as Biomedical (Biomed), Civil (Civ), Environmental (Env) or Mechanical (Mech), basic competence in both analog and digital theory is still necessary and expected. Thus, Circ1 is retained as a pre-requisite for Instrumentation \& Measurements, required for the Mech concentration, and Bioinstrumentation \& Measurements for the Biomed concentration. Moreover, working knowledge of analog and digital circuits should help students understand and develop techniques addressed in Manufacturing Processes required for Mech, Control Systems required for Biomed, Env and Mech, and Biomedical Engineering Design required for Biomed concentrations. Academic coursework aside, competence with both basic analog and digital circuits help students in all the other concentrations (Biomed, Civ, Env and Mech concentrations) communicate more knowledgeably and effectively with Elect and Comp students when interacting on multidisciplinary teams doing project work. Parameters of the multi-year multidisciplinary project work we require at Messiah College have been detailed in previous papers. ${ }^{1,2}$

Literature review and contribution of this work

Having established a rationale and place in the engineering curriculum for an electric circuit course that combines both analog and digital circuits, this section addresses related literature in the field of engineering education. A number of recent papers address improved methods of instruction for a course on electric circuits. Skromme, et al. have described the latest developments of a computer-based tutorial program involving a step-based approach to assist students in the learning key concepts of circuit analysis, resulting in significant gains for students over doing conventional textbook problems. ${ }^{3}$ Morrow recently reported on experience with implementing a blended-learning model involving more active learning, technology-enhanced exercises for an electric circuit course, resulting in improvement of student knowledge and satisfaction. ${ }^{4}$ Kolloffel \& de Jong have studied benefits of acquiring conceptual understanding facilitated by inquiry learning in a virtual lab, versus traditional instruction alone, concluding that students in the virtual lab condition did better. ${ }^{5}$ Ying and Morton have reviewed a number of teaching practices for teaching circuit analysis, including instructional videos to introduce lab equipment. ${ }^{6}$ Lawanto and Santoso investigated students' self-regulated learning while using enhanced guided notes as related to conceptual understanding of electric circuits. ${ }^{7}$ Rockland has described experience with flipping the circuit analysis course, including creating videos, structuring content, and assessing student learning. ${ }^{8}$ Several concept inventories ${ }^{9-12}$ have been developed to better assess students' understanding of circuits; Sangam and Jesiek analyzed four of these circuit concept inventories to assess their relative educational value ${ }^{13}$ including the ones used in our work. While these techniques may enhance the potential for student engagement and learning in the electric circuit course by improving on the traditional approach in significant ways, in this paper, we address a different equally important aspect; we propose a new nontraditional circuit course sequence that better fits the need of students in multidisciplinary 
engineering tracks, without adding another required engineering core course to an already full liberal arts / engineering curriculum. We report on our experience with the new circuits sequence here. The first course in the sequence uses strategic topic-selection and reordering to combine analog with digital in the introductory core course, better preparing all students in a multidisciplinary program for their engineering curriculum and future project work.

\section{Methods}

Creating and implementing the new introductory analog/digital electric circuits sequence from the previous Circuit Analysis and Digital Electronics courses involved reorganizing course content, creating a hybrid custom textbook, revising syllabus schedules and adjusting lab content. This section elaborates on practical decisions associated with this curriculum change and describes ways we have assessed students' learning and level of satisfaction.

Our approach in each of the two new courses has been to devote half the semester on analog circuits, and half on digital, for a roughly equal, balanced treatment. Starting with basic analog circuits follows the priority of historical precedence, but also is a practical approach in the lecture and in the lab, as it facilitates introducing overriding fundamentals such as ideal circuit elements, electrical quantities and essential test equipment in logical order. Thus, we settled on addressing analog circuits in the first half of Circ1 before digital circuits.

To divide the traditional treatment of Circuit Analysis into two halves, selected topics were chosen for fundamental exposure in Circ1, while the remaining concepts were deferred to Circ2. For example, in Circ1, attention is restricted to analyzing analog circuits with independent voltage and/or current sources only; circuits with dependent sources are left for Circ2. As a systematic approach to the analysis of analog circuits, students learn the Node Voltage Method in Circ1, but the Mesh Current Method is deferred to Circ2. Overall, the half-semester treatment of analog circuits ranges from source-resistor combinations with relevant laws and simplification techniques, to a section on Op Amp circuits, and concludes with an introduction to capacitors (C) and inductors (L). First (RC and RL) and second (RLC) order circuit behavior and analysis has been deferred to Circ2, except for a summary treatment of RC circuits in the Circ1 lab. Sinusoidal sources and the response of circuits in the frequency domain have also been moved to Circ2, along with phasor notation. Furthermore, students in Circ2 learn about transformers and how to compute instantaneous, average, and reactive power.

The half-semester treatment of digital electronic circuits for Circ1 includes digital theory as well as principles and practice of using common digital hardware (fixed logic) devices. Topical content includes comparison of digital versus analog quantities, number systems with operations and codes, digital logic gates with Boolean algebra, combinational logic circuits, functional logic devices, and a concluding introductory treatment of sequential logic devices for circuits. A fuller 
treatment of combinational and sequential logic circuits including latches, flip-flops, and counters is deferred to Circ2, as is the theory and application of Programmable Logic Devices. Memory devices, including Random Access Memory (static and dynamic) and Read Only Memory (various technologies including flash), and analog/digital conversion techniques are introduced in Circ2. Verilog was chosen to use both as an introduction to the idea of a Hardware Description Language and a tool for doing digital design and implementation in the laboratory. Multisim is also used extensively for simulating circuits prior to building them.

A search of the available texts revealed that it is easy to find separate analog and digital texts that cover all the material we wanted for both courses, but much harder to find one text for both courses or even one text for each course that combines analog and digital material in the way that we desired to teach them. So, we arranged with a publisher to have a custom textbook created for each course in the sequence. For Circ1, we retained the first seven chapters of Nilsson \& Riedel's Electric Circuits text for the analog portion, and combined that with six relevant chapters of Floyd's Digital Fundamentals: A Systems Approach. Both of these texts were available from Pearson, which facilitated the process. For Circ2, chapters 8, 9, and 10 of Nilsson $\&$ Riedel and chapters 7-10 and 12 of Floyd were combined to create a second custom text that covered the topics identified above. The choice of Floyd's Systems version of his digital texts was deliberate, as it includes a more complete treatment of the use of Hardware Description Language (HDL) to implement digital designs using programmable logic.

Adjustments in the lab for Circ1 (versus the former Circuit Analysis version) included selecting a smaller group of activities that support and explore analog circuit related material, and adding digital circuits activities at an appropriate level. For analog circuits, we use the first six prescribed experiments in Tsividis, A First Lab in Circuits and Electronics, and a one-period design challenge activity. An introduction to computer simulation for electric circuits using Multisim serves as a transitional activity between the analog and digital circuits sections. The lab schedule is concluded with four digital activities intended to help student learn the techniques of designing, constructing and testing combinational logic circuits with fixed logic devices.

For Circ2 lab, the first two labs followed experiments \#7 and part of \#8 in Tsividis. Four new lab experiments were created to emphasize and reinforce course material, including three that dealt with advanced topics in digital design and implementation.

Evaluating the extent to which students have learned essential concepts in the new analog/digital circuit course sequence consists of a work in progress for us. At the end of the Fall 2014 semester, we selected Circuit Concept Inventory (CCI available at www.foundationcoalition.org/ home/keycomponents/concept/circuits.html) item questions to assess the knowledge of key analog circuits concepts among students who completed Circ1 and Circ2. As for its appropriateness as a tool for evaluation, the CCI was included in a 2010 comparative analysis by 
Sangam and Jesiek. ${ }^{13}$ We have now migrated to the use of Determining and Interpreting Resistive Electric Circuits Concepts Test (DIRECT) ${ }^{12}$ at least for Circ1, since DIRECT has known statistical measures of quality, and is conveniently available as a pre- and post-test option with the web-based Circuit Tutor ${ }^{3}$ tool. The CCI assessment was given as a post-test to students in Circ1 on the last class period of the Fall 2014 semester, with the incentive of $1 \frac{1}{2}$ point extra credit applied to the final exam grade, for each CCI item answered correctly. The other CCI items selected for Circ2 students were given at the end of the semester, by verbal request of the instructor, with no extra incentive. Since a standard concept inventory for digital circuits does not appear to be available, we use selected questions of the final exam as a gauge of how well students learned those basics. The results of this assessment appear in the next section.

As an indication of student satisfaction with the new analog/digital approach for Circ1 and Circ2, some feedback by students in response to the open-ended questions, "What did you like 1) best or 2) least about this course?" were collected together with the usual Individual Development and Educational Assessment (IDEA) course evaluation surveys. Results of these responses relevant for this paper are also summarized in the next section.

During the spring 2015 semester, we plan to conduct a more formal survey of upper-class students addressing their perception of the new Circ1 course material and if or how it has helped them understand and communicate with other team members about digital circuitry associated with their project work. Results will be included in the final draft.

\section{Results}

\section{Circuits 1 Concept Assessment}

Overall, we have offered the new Circuits 1 (Circ1) course for six sections of approximately 2030 students each ( 150 students total), over the course of four semesters since Spring 2013. At the end of the Fall 2014 semester, a section of 20 Circ1 students took a post-test consisting of 21 items drawn from the CCI database that correspond with a subset of the topics addressed in the analog portion of that course. Based on the criterion of a 75-100\% majority with a correct response on one or more items, students appear to be proficient with the following Circ1 concepts:

1A. The magnitude of the equivalent series capacitance compared to individual values

1B. The relative magnitude of currents through resistors in a current divider

1C. Kirchhoff's Current Law \& current division at the junction of a series-parallel circuit

1D. Recognizing which of a combination of four resistors is connected in parallel, and which in series, for a Wheatstone Bridge configuration with bridge open or closed

1E. Configuration of a double SPDT switch combination required to close a circuit

1F. Kirchhoff's Voltage Law and voltage division by three unequal resistors to determine the lowest voltage 
1G. Pathway of current in a resistive circuit with a shorted branch

1H. Peak voltage, peak-to-peak voltage and period designations of a sine wave as given in a graphed example

Based on a slimmer majority of only $60-70 \%$ of students in the Circ1 class, students demonstrated a marginal proficiency on the following concepts:

1I. Dependence of the time constant in an $\mathrm{RC}$ circuit on the $\mathrm{R}$ and $\mathrm{C}$ values

1J. Kirchhoff's Voltage Law and voltage division by three unequal resistors to determine the highest voltage

$1 \mathrm{~K}$. The proper expression for the equivalent parallel-series combination of resistors in a

Wheatstone Bridge configuration with the bridge closed (shorted)

1L. The average voltage of a sine wave as given in a graphed example

Based an unacceptable minority of only $15-35 \%$ of the class getting the correct response, students mostly misunderstood the following Circ1 concepts:

$1 \mathrm{M}$. The relative magnitude of voltages across individual capacitors in a series capacitance combination

1N. Dependence of the capacitor voltage in an $\mathrm{RC}$ circuit on the charging time, in time constants

Selected items from the Fall 2014 final exam show the proficiency of Circ1 students with concepts in the digital part of the course. The first example (1O) involves a four-input variable logic function expressed in Boolean form, based on given Truth Table values. For this function, students were asked to propose a digital circuit implementation in three different forms: a) showing connections between standard available logic gates, b) using a 16 to 1 Multiplexer (MUX), and c) employing an 8 to 1 MUX. Each part was worth 5 points. The mean score on this section 12.5 out of 15 points, while the median was 13.75. As the class average was above $80 \%$, we can consider this result to indicate satisfactory proficiency overall. A second example (1P) involves the behavior of a sequential logic element: the JK flip-flop (FF). Given a diagram of the JK FF with a negative-edge triggered clock input, and a timing diagram showing the pulse train waveforms for the clock, J and K inputs, the student was to predict the output of the JK FF. On this item, students scored an average of 4.35 out of 5 points. Again, the average above $80 \%$ appears to indicate satisfactory proficiency.

Circuits 2 Concept Assessment

The new Circuits 2 (Circ2) course was offered for the first time in the Spring of 2014 and was taken by 9 students. Of these 9 students, seven took a post-test consisting of 17 items drawn from the CCI database at the end of Fall 2014. Based on the criterion of a 71-100\% majority with a correct response on one or more items, students appear to be proficient with the following Circ 2 concepts: 
2A. Given an AC input voltage in graphical form, the output of a dependent voltage source circuit

2B. The average, peak, peak-to-peak, and rms voltages of a sine wave as in graphed example

2C. Equivalent circuit of an RLC series combination connected to an AC voltage source at resonance

2D. Graphical representation of an underdamped response as a plot of $v(t)$ versus $t$

2E. Initial conditions for an RL step response circuit before and after the switch is closed

2F. Graphical representation of $i(t)$ versus $t$ for an RL step response circuit

2G. Time constant for an $\mathrm{RC}$ step response circuit in terms of $\mathrm{R}$ and $\mathrm{C}$

$2 \mathrm{H}$. Dependence of the capacitor charging time for an $\mathrm{RC}$ circuit on the time constant $(\tau)$

Based an unacceptable minority of only $29-57 \%$ of the class getting the correct response, students mostly misunderstood the following Circ2 concepts:

2I. Given an AC input voltage in graphical form, the output of a dependent current source circuit

2J. Relative magnitude and phase of R, L and C voltages in graphical form for an RLC series circuit at resonance

$2 \mathrm{~K}$. Graphical representation of an overdamped response as a plot of $\mathrm{v}(\mathrm{t})$ versus $\mathrm{t}$

2L. Dependence on $\tau$ of capacitor charging time to effective completion for an RC circuit

Selected items from the Fall 2014 Circ2 final exam show the proficiency of students with concepts in the digital part of the course. The first example (2M) asked students to design of three-bit asynchronous counter with a truncated count sequence. The question also asked them to explain what would happen if the counter were to power up in a condition other than one of the valid states. The design and question were worth a total of 5 points. The mean score was 4.13 , while the median was 4.5 . The average being above $80 \%$ indicates satisfactory proficiency. A second example $(2 \mathrm{~N})$ asked students to discern the function and purpose of an undocumented Verilog module. The result was a mean score of 3.75, with a median of 4. A third example (2O) asked students to design a BCD validity checking circuit using a 1-to-16 demultiplexer. This question probed the students' ability to use a digital integrated circuit in a slightly unusual way. The mean score on this question was 4.13 , with a median of 5 , which shows that the majority of the students were able to execute the design without difficulty.

Section IV provides a more complete analysis and discussion of the misconceptions students had that led to erroneous choices on the above assessments.

Circuits 1 Student Feedback

As an indicator of student satisfaction, at the end of the Spring and Fall 2014 semesters, 49 Circ1 student surveys included the questions "What did you like 1) best or 2) least about this course?" Of these 49 students, without prompting, 15 mentioned the digital part (or labs) in some way as what they liked best, while 10 students mentioned analog (e.g., its difficulty to grasp, homework 
or labs) as what they liked least. None of the responses went the other way (i.e., preference for analog over digital). Since the unprompted student preference for digital over analog was by far the most frequently mentioned specific in these responses, we take the opinion expressed to be significant one and fairly widespread.

Circuits 2 Student Feedback

At the end of the Fall 2014 semester, the first class of 9 students taking the course were asked to complete survey questions including "What did you like 1) best or 2) least about this course? and 3) What suggestions would you make to improve this course?" Of the 8 students who took the time to respond, 4 mentioned the course material specifically as the part they liked best, 3 mentioned the lab exercises, and 2 referred to the digital material. In response to the question of what they liked least, 3 said that they found the tests hard and 2 mentioned the labs (some of the new labs had bugs). Most of the responses to the question of how to improve the course were indicated that the students thought the tests were too hard, which is not an unexpected response to this type of question.

\section{Analysis and Discussion}

\section{Circuits 1 Misconceptions}

Using Circuits Concept Inventory (CCI) items or other final exam questions can help assess how well students have learned essential aspects of the subject material, but also can reveal where misconceptions remain. An incorrect response on one of these items can reflect the deficiency of individual students, or if it wider spread among a majority of students in the class, a deficiency in the teaching method that needs to be addressed. Focusing first on the poorest results with CCI items from the previous section reveals the following Circ1 misconceptions on the analog side:

M1A. Voltages split across capacitors in series with a voltage source just like they do across resistors in series ( $60 \%$ of students)

M1B. Inaccurate view of how the progress toward completion of the exponentially charging voltage in an RC circuit depends on the time constant (85\% of students)

All other Circ1 misconceptions on the analog side, as revealed by distractor options of the multiple choice items on this particular assessment, occurred at a rate of only $20 \%$ or less.

The widespread misconception of M1A resulted from insufficiently detailed treatment of capacitor combination circuit behavior in the Circ1 course. Students learned to calculate the equivalent capacitance of circuit networks made up of multiple capacitors exclusively in series, parallel or hybrid series/parallel combinations. However, they were not asked in this course to determine how the voltages split up across capacitors in a network (e.g. series) connected to a voltage source, as they were required to do with a series resistance (e.g. voltage divider) network. While they were expected to understand that the same amount of electric current 
passes through each and every one of the resistors in a purely series resistor circuit, they did not recall or apply here the relationship between charge and voltage across a capacitor $(\mathrm{Q}=\mathrm{CV})$, that they should have learned in physics, and from which the important capacitor current circuit relationship $(\mathrm{i}=\mathrm{CdV} / \mathrm{dt})$ is derived. Had they understood that each capacitor in a series capacitance circuit holds the same amount of charge (equal and opposite on each plate), they could have easily applied the inverse proportionality $(\mathrm{V}=\mathrm{Q} / \mathrm{C})$ to realize that the smallest capacitor experiences the greatest potential difference, and vice versa. As it was, they conflated the behavior of the series capacitance circuit with that of the series resistance circuit, as far as voltage division is concerned. This can be addressed in the course by giving students assigned examples that not only ask them compute equivalent capacitance of a multiple capacitance network, but also in some simple examples work step by step through the reasoning to determine the voltage across each, based on a known DC voltage applied to the network.

The widespread misconception of M1B can be explained by insufficient practice in Circ1 with the analytical aspects of the exponential charging function of an RC circuit. While RC circuit behavior is addressed in the lab experimentally, including an introductory treatment of the theory by the instructor, detailed analysis of the RC combination as a first order circuit is left for Circ2. However, in Circ1, we expose students in all branches of engineering to $\mathrm{RC}$ circuit behavior, and want them to have a clear concept to guide their intuition. Getting a better idea for the role of the $\tau=\mathrm{RC}$ time constant might be facilitated by asking them to plot the graph of the charging and/or discharging capacitor voltage with marks along the time axis indicating the number of time constants that have passed. A reflective follow up question asking students to consider relative progress of the capacitor toward the "steady state" completion of full voltage after $0,1,3,5$, etc. time constants might help drive home this concept more clearly and accurately.

As for proficiency of Circ1 students on digital theory, some observations can be made about common errors made on final exam items. On the digital item 10 , the most common error among students who lost credit was to connect select inputs of the MUX to input variables in reverse order. As for $1 \mathrm{P}$, the most common error was failure to correctly distinguish negative versus positive edge triggering, or edge versus level sensitive operation. Overall, misconceptions on the basic digital theory among students in Circ1 were generally not widespread as on the misconceptions associated with basic analog circuit theory.

Circuits 2 Misconceptions

Among students with electrical or computer concentrations, the following Circ 2 misconceptions on the analog side are revealed, in each case at a rate of $43 \%(4 / 7)$ of the students:

M2A. No phase inversion recognized in the linkage between a dependent AC current source and the original source on which it depends, when the current direction arrows are opposite M2B. For an RLC series circuit at resonance, no voltage appears across the capacitor or inductor individually 
$\mathrm{M} 2 \mathrm{C}$. In a plot of $\mathrm{v}(\mathrm{t})$ versus $\mathrm{t}$, an over-damped response looks like simple 1st order exponential decay

All other Circ2 misconceptions (14) on analog material, as revealed by distractor options of the multiple choice items on this particular assessment, occurred at a rate of $29 \%(2 / 7)$ or less.

Misconception M2A may have to do as much with recognizing and interpreting the symbolism of dependent source circuits, as much as anything else. Since the current dependent current source of the circuit was created in Multisim, it assumes familiarity with the dependent current source representation in that format. The circuit also requires students to recognize significance of current direction in the dependent source, as compared to the original, so as to realize the phase inversion it has at the output. More practice for the students with dependent current source representations, such as in Multisim, and examples of phase inversion may help here.

Misconception M2B reveals a deficiency in students understanding of how phase cancellation occurs in an RLC circuit at resonance. To address this issue, using an RLC circuit at resonance in the lab, it may be helpful to have students measure the AC voltage of the capacitor and the inductor individually on the oscilloscope, to see that they are equal in magnitude and 180 degrees out of phase, rather than zero in each case. Furthermore, it may help students to observe what happens to these voltages compared to each other when the frequency of the source is adjusted somewhat above and below the resonance frequency.

Misconception M2C indicates that students have a tendency to confuse the voltage response of a $2^{\text {nd }}$ order over-damped RLC circuit with that of a $1^{\text {st }}$ order circuit. Students may need more practice in creating and analyzing the $1^{\text {st }}$ and $2^{\text {nd }}$ order voltage response curves in order to gain enough familiarity to distinguish between them, by recognizing critical features.

Regarding the results of item $2 \mathrm{~N}$ reported in results, Verilog was the hardware description language used for numerous examples all semester in Circ2, including a lab exercise; students were expected to recognize the very familiar D-type flip-flop implemented by the module. However, many students missed the slight twist of it being negative-edge triggered and so many lost one of the 5 possible points. The average being slightly below the $80 \%$ threshold means technically below satisfactory proficiency, but based on the median we would conclude that the reason had more to do with attention to detail than with an understanding of digital theory.

Commentary on Student Feedback

The results of Circ1 student surveys, though anecdotal, indicate a fairly strong and widespread preference for learning digital versus analog circuits. Recognizing that most of these students choose concentrations other than electrical or computer, this result suggests that many multidisciplinary students like the new version of the Circ1 course better than they would the traditional version including purely analog circuits. While such a reaction was not the original reason for making this change, it indicates that students are ready and willing, even eager, to 
address the need in their own engineering education for the kind of competency that is becoming required in all disciplines.

The student surveys for Circ2 are few in number, but there seems to be general consensus that the course material is relevant. Student enthusiasm for the digital side was more apparent in class and lab than in the survey results, but this is difficult to measure. We intend to again evaluate Circ2 in the Spring of 2015; with a class size of 19, the results should be more informative.

\section{Conclusions and Future Work}

This paper has focused on a core course for a multidisciplinary engineering curriculum that combines analog and digital circuits; content formation, sequence considerations, student proficiency with essential concepts, and student feedback at our institution has been highlighted to indicate the extent that this curriculum innovation has been successful in our experience. Future work needs to address student misconceptions, and to measure whether students perceive the benefits of broader exposure in electric circuits when doing later course and project work.

The authors intend to develop and conduct a more formal survey of student opinions during the Spring 2015 semester. The instructor of Circuits 1 has incorporated the Circuit Tutor ${ }^{3}$ for the Spring 2015 semester to help bolster students' learning of basic analog concepts, and is in the process of developing concept questions for more engaged in-class active-learning that span both the analog and digital parts of the course. Although Concept Inventory surveys (e.g., CCI and DIRECT $^{12,13}$ for pre- and post-test diagnostics) exist for analog circuits concepts, a similar concept inventory for digital circuits does not appear to be available, and needs to be developed.

For those who wish to try this non-traditional approach, it should be understood that a high level of cooperation between instructors of the traditional analog circuits and digital electronics courses, and a few semesters of adjustment is necessary to accomplish the transition. Beyond the difficulty of those challenges, we recommend the new approach as very worthwhile, particularly for students who are likely to complete their engineering degree with no formal background in electrical theory other than Circuits 1.

\section{Acknowledgments}

The authors would like to thank Dr. Randall Fish for suggesting the combined Digital and Analog Circuits approach. 


\section{References}

1. Underwood, H and D. Pratt, "Competitive Placement of Engineering Students on Multiyear Project Teams," Proceedings of the 2014 ASEE Annual Conference, Indianapolis.

2. Underwood, H., "Using the Portfolio Approach to Assess Multi-year Engineering Projects: A Case Study," Proceedings of the 2013 ASEE Annual Conference, Atlanta.

3. Skromme, B. et al., "Expansion and Evaluation of a Step-based Tutorial Program for Linear Circuit Analysis," Proceedings of the 2014 ASEE Annual Conference, Indianapolis.

4. Morrow, M., "Experiences with Electric Circuit Analysis in a Blended Learning Model," Proceedings of the 2014 ASEE Annual Conference, Indianapolis.

5. Kolloffel, B. and de Jong, T., "Conceptual understanding about electric circuits in secondary vocational engineering education: Combining traditional instruction with inquiry learning in a virtual lab," Journal of Engineering Education, Volume 102, pages 375-393.

6. Ying, L. and Morton, T., Novel Practices in Teaching Circuit Analysis in an EET Program," Proceedings of the 2013 ASEE Annual Conference, Atlanta.

7. Lawanto, O. and Santoso, H., Investigating Students' Self-Regulated Learning While Learning Electric Circuit Concepts with Enhanced Guided Notes," Proceedings of the 2013 ASEE Annual Conference, Atlanta.

8. Rockland, R., et al., Learning outside the classroom - Flipping an Undergraduate Circuits Analysis Course," Proceedings of the 2013 ASEE Annual Conference, Atlanta.

9. Sangam, D. and Jesiek, B., "Conceptual Understanding of Resistive Electric Circuits Among First-Year Engineering Students," Proceedings of the 2012 ASEE Annual Conference, San Antonio.

10. Ogunfunmi, T. and Mahmudur, R., Concept Inventory Assessment Instruments for Circuits Courses, Proceedings of the 2011 ASEE Annual Conference, Vancouver.

11. Rahman, M. and Ogunfunmi, T., "A set of questions for a concept inventory for a DC Circuits course," Proceedings of 2010 IEEE International Symposium on Circuits and Systems (ISCAS), Paris.

12. Englehardt, P. and Beichner, R., Students' understanding of direct current resistive electrical circuits, American Journal of Physics, Volume 72, Number 1, January 2004.

13. Sangam, D. and Jesiek, B., "Circuit Concept Inventories: A Comparative Analysis." Proceedings of the 2010 ASEE Anuиal Conference, Louisville, KY. 